\title{
METHODOLOGY FOR HIGH-FIDELITY DETERMINISTIC MODELLING OF SWISS LWR FUEL ASSEMBLIES
}

\author{
A. Bernal, M. Pecchia, D. Rochman, A. Vasiliev and H. Ferroukhi \\ Laboratory for Reactor Physics and Thermal-Hydraulics, Paul Scherrer Institut. \\ Forschungsstrasse 111, 5232 Villigen PSI, Switzerland. \\ alvaro.bernal@psi.ch,marco.pecchia@psi.ch,dimitri-alexandre.rochman@psi.ch, \\ alexander.vasiliev@psi.ch,hakim.ferroukhi@psi.ch
}

\begin{abstract}
The main goal of this work is to perform pin-by-pin calculations of Swiss LWR fuel assemblies with neutron transport deterministic methods. At Paul Scherrer Institut (PSI), LWR calculations are performed with the core management system CMSYS, which is based on the Studsvik suite of codes. CMSYS includes models for all the Swiss reactors validated against a database of experimental information. Moreover, PSI has improved the pin power calculations by developing models of Swiss fuel assemblies for the Monte Carlo code MCNP, with the isotopic compositions obtained from the In-Core Fuel Management data of the Studsvik suite of codes, by using the SNF code. A step forward is to use a neutron code based on fast deterministic neutron transport methods. The method used in this work is based on a planar Method of Characteristics in which the axial coupling is solved by 1D SP3 method. The neutron code used is nTRACER. Thus, the methodology of this work develops nTRACER models of Swiss PWR fuel assemblies, in which the fuel of each pin and axial level is modelled with the isotopic composition obtained from SNF. This methodology was applied to 2D and 3D calculations of a Swiss PWR fuel assembly. However, this method has two main limitations. First, the cross sections libraries of nTRACER lack some of the isotopes obtained by SNF. Fortunately, this work proves that the missing isotopes do not have a strong effect on $k_{e f f}$ and the power distribution. Second, the 3D models require high computational memory resources, that is, more than $260 \mathrm{~Gb}$. Thus, the nTRACER code was modified, so now it uses only 8 $\mathrm{Gb}$, without any loss of accuracy. Finally, the $k_{e f f}$ and power results are compared with Monte Carlo calculations obtained by Serpent.
\end{abstract}

KEYWORDS: PWR, SNF, nTRACER, deterministic method

\section{INTRODUCTION}

An important goal at Paul Scherrer Institut (PSI) is to conduct research aimed at developing stateof-the-art computational methodologies and incorporate them into a consistent Code System for integral multi-physics multi-scale LWR safety analyses. The core management system of PSI, CMSYS [1], is based on the Studsvik suite of codes. This system includes models for all the Swiss reactors up to the latest operated cycles, which are validated against a database of experimental information. These models are used for lattice calculations with CASMO [2], as well as full core 
calculations with SIMULATE [3]. Moreover, the SNF code [4], which belongs to the Studsvik suite of codes, was recently added to calculate the isotopic compositions and radiation sources of different Swiss fuel assemblies [5]. This code makes use of the In-Core Fuel Management (ICFM) data of CASMO/SIMULATE, providing detailed information of the state of the fuel and its operating history.

This sequence was used at PSI to define 3D isotopic compositions of MCNP models of Swiss fuel assemblies, to perform high-fidelity Monte Carlo calculations, such as criticality studies of spent nuclear fuel and intra-pin power distributions studies in fuel assemblies [6]. A step forward in this research is the extension of the previous sequence to enhanced-resolution deterministic calculations. Although Monte Carlo calculations provide accurate results, they have two main drawbacks. First, the high computational time to obtain low statistical errors. Second, in practice they are limited to steady state calculations, though there are some approaches for transient and fuel depletion calculations [7]. By contrast, deterministic calculations might be faster and can solve the time-dependent Neutron Transport Equation with different methods.

Among the different deterministic methods, the methodology applied in this work employs a 2DMOC/1D-nodal method to obtain accurate results with competitive computational times [8]. This method is based on a planar Method of Characteristics (MOC) in which the axial coupling is solved by 1D nodal methods, such as P1 or SP3 methods. At PSI, the 2D/1D neutron code used is nTRACER [9], developed at the Seoul National University. Although nTRACER can perform Core Follow calculations [10], the goal at PSI is to perform consistent calculations with the validated database of CMSYS. For this reason, the methodology developed in this work consists of three main steps. First, ICFM calculations with CASMO/SIMULATE to provide the isotopic and power history data to SNF, which are explained in Section 2.1. Second, isotopic calculations with SNF to provide the compositions to nTRACER, which are described in Section 2.2. Third, neutronics calculations with nTRACER, which are summarised in Section 2.3. However, this method has two main problems. First, the cross sections libraries of nTRACER lack some of the isotopes obtained by SNF. Second, the 3D models require high computational memory resources, that is, more than $260 \mathrm{~Gb}$. Thus, the nTRACER code was modified, so now it uses only $8 \mathrm{~Gb}$. Further details of this modification are given in Section 2.3.

The methodology of this work was applied to 2D and 3D calculations of a Swiss PWR fuel assembly, and it was verified with Monte Carlo calculations with Serpent [7]. This verification consists in a code-to-code comparison of $k_{e f f}$ and the power distribution, as shown in Section 3. The outline of the rest of the paper is as follows. Section 2 explains the methodology of this work. Section 3 describes the application case, it shows the results, and it proves that the missing isotopes in the nTRACER libraries do not have a strong effect on $k_{\text {eff }}$ and the power distribution. Section 4 summarises the conclusions.

\section{METHOD}

\subsection{In-Core Fuel Management Calculations with CASMO/SIMULATE}

This method was already applied in [5] and consists in calculating validated models of Swiss PWRs by using CASMO5 and SIMULATE3 for the whole reactor. These validated models come from the core management system of PSI, CMSYS, which is based on: realistic irradiation history, core 
characteristics, assembly characteristics and loading patterns. The method has two main strengths. First, all the previous parameters are obtained from the plant operation. Second, the results obtained with CASMO/SIMULATE are validated not only against the in-core measurements, but also against Post Irradiation Examination data (using the SNF code).

\subsection{Calculation of the Isotopic Compositions of the Fuel Assemblies with SNF}

This method uses the version 1.6.4 of the SNF code to calculate the isotopic concentrations of certain fuel assemblies and fuel pins from the CMSYS database. As explained in [4], SNF takes into account any radial or axial heterogeneity in the analysed assemblies, based on the calculations from the In-Core Fuel Management with CASMO/SIMULATE, as mentioned in Section 2.1. CASMO5 generates the nuclear data, whereas SIMULATE3 provides a detailed power history of the fuel assemblies and pins. Moreover, SIMULATE3 also accounts for effects caused by changes in the following parameters: fuel temperature, coolant density, boron concentration and control rods. From the SNF calculation, one obtains different isotopic concentrations for each pin and axial segment of each assembly, though in this work only one fuel assembly was considered. However, it should be highlighted, that SNF does not print the oxygen concentration of the fuel. In addition, SNF calculates the concentrations for different cooling times, but in this work the authors chose a cooling time of 0.1 days, to model the current fuel composition in the core.

\subsection{Neutronics Calculations with nTRACER}

This section describes three different processes. First, the automatic generation of the model for nTRACER, from the isotopic composition and geometry of CASMO/SIMULATE/SNF. Second, the modification of nTRACER to reduce the computational memory requirements, because of the modelling of a large number of pins with different isotopic concentrations. Third, the actual calculation with nTRACER. For the first step, a Fortran code was developed with the next capabilities:

- Reading the isotopic concentrations from the SNF outputs (fission products and actinides) for all the fuel pins and axial nodes of a certain fuel assembly.

- Reading the oxygen and burnable poisons concentrations of the fuel pins, from the CASMO5 outputs defining the axial nodes of the SIMULATE3 model.

- Writing the material composition of each fuel pin and axial node for the nTRACER input.

- Writing a list of the isotopes of these compositions which are not present in the nTRACER libraries, for each pin and axial node, and including the concentrations of these isotopes.

- Writing a MATLAB file containing the isotopic concentrations for each pin and axial node.

Unfortunately, this enhanced material modelling is limited in the version of nTRACER (2.50) used in this work. The reason is that nTRACER performs the ray tracing of each cell defined in the input, and one needs to define one cell for each pin and axial segment, since they contain different isotopic concentrations. Therefore, for a typical PWR fuel assembly, this process not only requires a lot of computational time, but also runs out of memory. However, the cell ray tracing only depends on the geometry of the cell. Actually, once the ray tracing is performed, nTRACER 
assigns to each region inside a pin and axial segment its corresponding material, that is, the isotopic concentrations.

To overcome this problem, the authors changed the source code of nTRACER. In this new version (2.50.PSI), one can define only few cells composed of certain regions, and then one can define the materials for these regions in each pin and axial segment of each fuel assembly. Actually, in this work, one only needs to define three cells for the following pins: fuel, guide tube and reflector. To fill the material composition for each pin, axial segment and fuel assembly, one just has to add the following line in the input: mixcell ixa iya ix iy iz $M_{1} \cdots M_{N}$; where ixa is the X position of the assembly in the core, iya is the Y position of the assembly in the core, ix is the $\mathrm{X}$ position of the pin in the assembly, iy is the Y position of the pin in the assembly, $i z$ is the axial segment, and $M_{i}$ is the material of the region $i$ of the pin. It was checked that this modification does not induce differences in the results.

Finally, nTRACER solves several calculations of 2D MOC and 3D Coarse Mesh Finite Difference (CMFD). In addition, the $\mathrm{SP}_{3}$ nodal method is used in the 3D CMFD calculations, which provides the axial nodal flux distribution, to use coarse axial mesh sizes with enough accuracy. The 2D MOC obtains the flux distribution with pin level details for each axial plane, where each axial plane is coupled with the adjacent ones through the axial leakage terms. Then, this flux distribution is used to generate cell-homogenized cross sections for solving the 3D CMFD problem. Once it is solved, the solution is used to update the fission source distribution and the axial leakage terms for the 2D MOC problems. This iteration process is followed until convergence is reached. Further details of the numerical methods are given in [9].

\section{RESULTS}

The method was applied to a fuel assembly, of a specific cycle of a Swiss PWR, which is described in Section 3.1. The results are $k_{e f f}$ and the power distribution of the steady state calculations, 2D and 3D. These results are evaluated by means of the relative errors of Eqs. (1) and (2). The boundary conditions are reflective for the north, south, east and west boundaries. As regards the top and bottom boundary conditions, they are vacuum for the 3D calculation, and they are reflective for the 2D calculation. The evaluation of the results consists in a code-to-code comparison of nTRACER (version 2.50.PSI) and Serpent (version 2.1.30). Section 3.2 defines the main characteristics of the models of these codes. Finally, Sections 3.3 and 3.4 show the results for $2 \mathrm{D}$ and 3D calculations.

$$
\begin{gathered}
k_{\text {eff }} \text { relative error }(\mathrm{pcm})=\left(k_{e f f}-k_{e f f_{r e f}}\right) / k_{\text {ef } f_{r e f}} \cdot 10^{5} \\
\text { Power relative error }(\%)=\left(\text { Power }- \text { Power }_{r e f}\right) / \text { Power }_{r e f} \cdot 100
\end{gathered}
$$

\subsection{Description of the Application Case}

Fig. 1a shows the fuel assemblies map of the Swiss PWR, and the selected fuel assembly with black color. This fuel assembly is composed of $15 \times 15$ pins and it was burnt in previous cycles. Fig. 1b displays the burnup (MWd/kg) of the fuel assembly. The geometry and materials of the fuel assembly come from CMSYS. The fuel assembly contains 20 guide tube pins composed of three regions: water inside the guide tube, the guide tube and the water outside the guide tube. Regarding 
the fuel pins, they are composed of 4 regions: fuel, gap, cladding and water. In addition, each pin is divided in 40 axial levels of $8.95 \mathrm{~cm}$ in length. The fuel enrichment was $1.9 \%$ for the first two axial levels, and $3.5 \%$ for the rest. Moreover, two additional axial levels are considered for the bottom and top reflector, with the same length. The top reflector also contains the guide tubes. On the other hand, for the 2D calculation, the axial level 31 was selected, but the axial length was set to $1.0 \mathrm{~cm}$. Regarding the temperatures, SIMULATE calculated the fuel temperature in the fuel assembly for each axial level, which is displayed in Fig. 1c. By contrast, the same temperature $(574.70 \mathrm{~K})$ and density $(0.72 \mathrm{~g} / \mathrm{cc})$ of the moderator were used for all the axial levels. The same thermal-hydraulic values were used in Serpent and nTRACER.

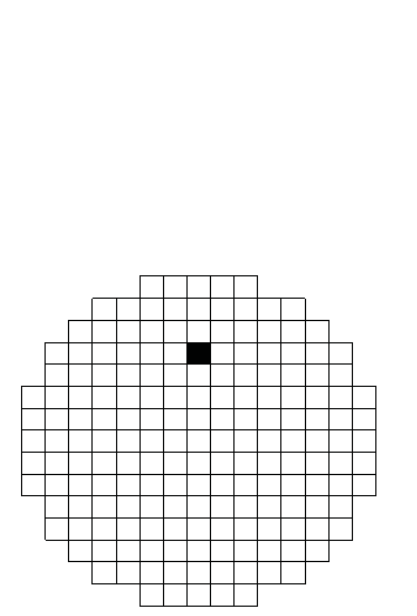

(a) Position of the FA in the core.

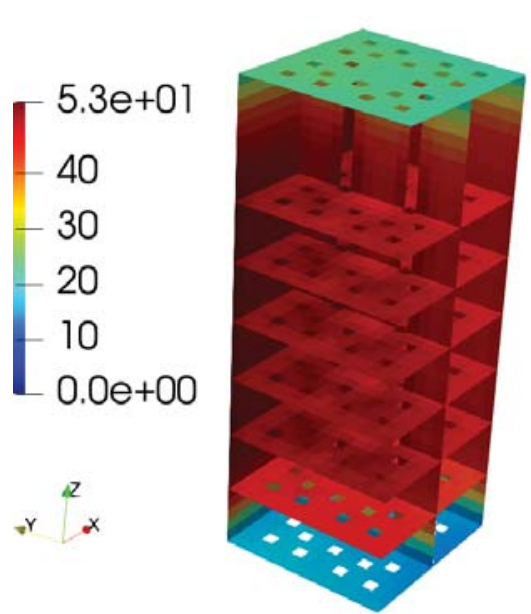

(b) Burnup of the FA (MWd/kg).

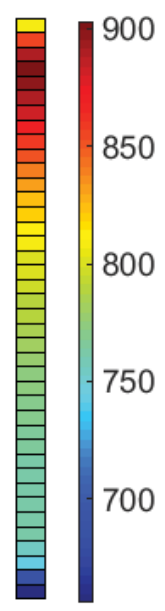

(c) Axial fuel temperature (K).

Figure 1: Description of the application case

\subsection{Description of the Models}

The main characteristics of the Serpent models are the following. The number of neutrons per generation is 1000000 and the number of inactive generations is 50. The number of active generations is 500 for $2 \mathrm{D}$ and 300 for $3 \mathrm{D}$. As regards the ACE files, the authors used the ACE files included in Serpent for the ENDF/B-VII.0 library, including the thermal scattering data for $\mathrm{H}$ in $\mathrm{H}_{2} \mathrm{O}$. However, these ACE files lack 172 isotopes (fission products and actinides) obtained by SNF. The complete list is not given in this paper, but examples of these isotopes are: Fe-55, Ni-63, Ge-77, Np-240 and Pa-234. For all these isotopes, the authors obtained the ACE files from the TENDL-2017 library [11], by processing this library with NJOY2016 [12]. This processing is based on the method proposed in [13].

The main characteristics of the nTRACER models are the following. In all the calculations, nTRACER calculated the effective cross sections of all the resonance isotopes included in the cross section libraries, by using the subgroup method; one can find further details of the method in [9]. As regards the ray parameters, the ray spacing was $0.05 \mathrm{~cm}$, the number of azimuthal ray angles was 128 and the number of polar angles was 6 . Regarding the scattering source, the models use Legendre polynomials up to order 3. Regarding the cross sections libraries, the version 2.50 of nTRACER uses a multigroup library and a resonance library, both based on ENDF/B-VII.0 and 
processed with NJOY2016. The libraries use 47 energy groups and they can calculate the resonance parameters for 68 nuclides (55 for fuel and 13 for cladding). However, the libraries lack 188 isotopes obtained by SNF, similar to those mentioned in the previous paragraph.

\subsection{D Results}

The $k_{\text {eff }}$ results for the Serpent calculations are $0.97711 \pm 0.00003$ for the case with all the isotopes of SNF, and 0.97794 \pm 0.00003 for the case with the isotopes of SNF included in the nTRACER libraries. Considering as reference the case with all the isotopes, the missing isotopes give a $k_{e f f}$ relative error of $84.94 \mathrm{pcm}$. Regarding the power results, the maximum relative uncertainty of the power due to Monte Carlo calculations is $0.07 \%$. In addition, Fig. 2 displays the power relative errors due to the missing isotopes, showing a maximum value about $0.25 \%$. From these results, one can conclude that the effect of the missing isotopes is not significant in the Serpent calculations.
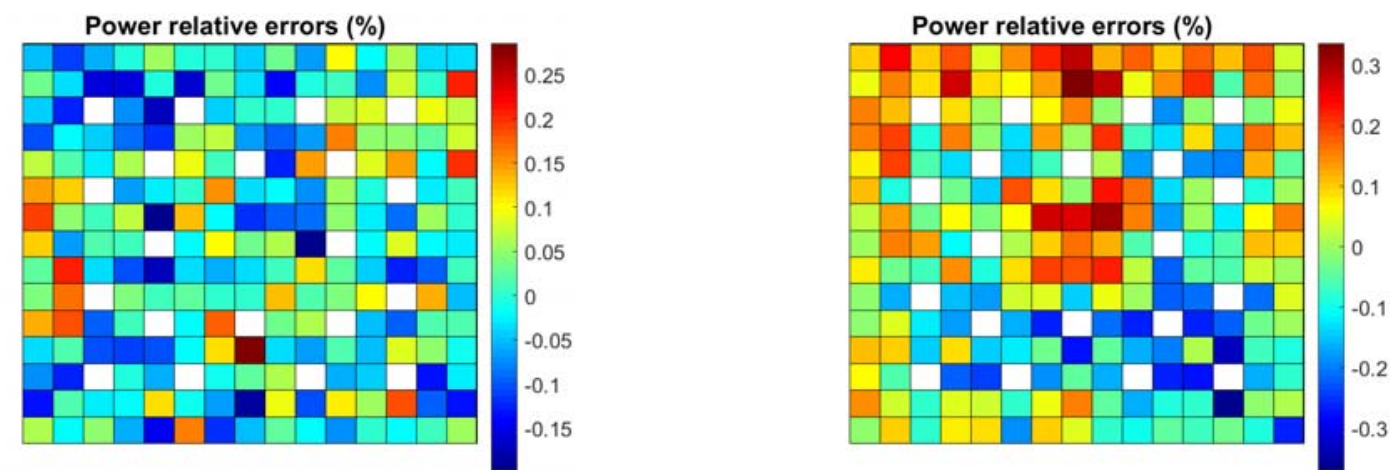

Figure 2: Effect of the missing isotopes on the power. Figure 3: 2D results for nTRACER.

For nTRACER, the $k_{\text {eff }}$ is 0.98175 . Considering as reference the Serpent case with the same isotopes, the $k_{e f f}$ relative error is $389.59 \mathrm{pcm}$. As regards the power results, Fig.3 shows the power relative errors for nTRACER, displaying a maximum value about $0.3 \%$. From these results, one can conclude that the results show good agreement. On the other hand, the computational times, with 12 OMP threads, were the following: 22086 s for the Serpent calculation with the isotopes included in the nTRACER libraries; $196 \mathrm{~s}$ for the nTRACER calculation.

\subsection{D Results}

The $k_{\text {eff }}$ results for the Serpent calculations are $0.98232 \pm 0.00008$ for the case with all the isotopes of SNF, and $0.98297 \pm 0.00004$ for the case with the isotopes of SNF included in the nTRACER libraries. Considering as reference the case with all the isotopes, the missing isotopes give a $k_{e f f}$ relative error of $66.17 \mathrm{pcm}$. Regarding the power, the maximum relative uncertainty of the power due to Monte Carlo calculations is $1.75 \%$. On the other hand, the $k_{e f f}$ for nTRACER is 0.98709. Considering as reference the Serpent case with the same isotopes, the $k_{e f f}$ relative error is $419.14 \mathrm{pcm}$. As regards the power, Fig. 4 displays the axial power for the different cases, whereas Fig. 5 shows the axial power relative errors. These figures show two additional calculations of nTRACER: one with the double number of axial levels and other with the double number of polar angles. In Fig. 5, the reference solution for "Missing isotopes" is the Serpent calculation with all 
the isotopes; for the nTRACER results, the reference solution is the Serpent calculation with the isotopes included in the nTRACER libraries. In addition, the computational times, with 6 MPI tasks and 12 OMP threads, were the following: $175130 \mathrm{~s}$ for the Serpent calculation with the isotopes included in the nTRACER libraries, $4391 \mathrm{~s}$ for the nTRACER calculation.

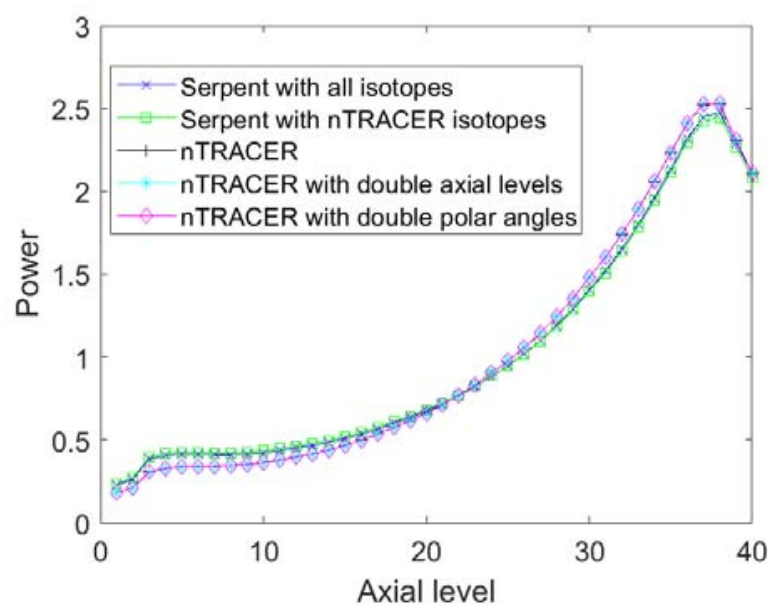

Figure 4: Axial power for 3D results.

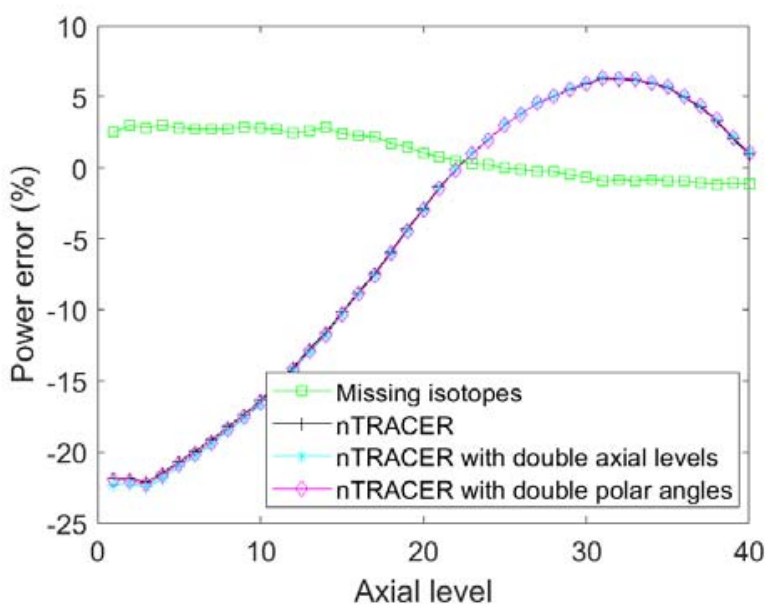

Figure 5: Power relative errors for 3D results.

According to these results, three conclusions are drawn. First, the effect of the missing isotopes is not significant. Second, the increase of the axial levels and polar angles does not have a significant effect on the power results. Third, the results show good agreement, but there are significant power relative errors at the bottom axial levels. This power tilt could be due to the cross section libraries or the resonance treatment of nTRACER.

\section{CONCLUSIONS}

This work has allowed to test a methodology based on SNF/nTRACER to do 3D full core transport calculations (pin-by-pin) with frozen feedback/burnup. This methodology consists of three steps. First, ICFM calculations with CASMO/SIMULATE to provide the isotopic and power history data to SNF. Second, isotopic calculations with SNF. Third, neutronics calculations with nTRACER using the isotopes obtained by SNF. Although this method could not be used with the original version of nTRACER because it run out of memory (more than $260 \mathrm{~Gb}$ ), the authors modified the source code of nTRACER limiting the memory to $8 \mathrm{~Gb}$.

The methodology of this work was applied to 2D and 3D calculations of a fuel assembly, of a specific cycle of a Swiss PWR. The results are based on $k_{\text {eff }}$ and the power distribution. Two issues were analysed. First, the effect of missing isotopes in the nTRACER libraries. This was done by comparing two simulations with the Serpent code: one with all the isotopes and other with only the isotopes included in the nTRACER libraries. Second, the methods and cross section libraries of nTRACER. This was done by means of a code-to-code comparison with the Serpent code. Three conclusions are drawn. First, the effect of the missing isotopes is negligible. Second, the $2 \mathrm{D}$ results show good agreement, but the 3D case shows significant relative power errors at the bottom axial levels. Third, nTRACER is much faster than Serpent. As future work, two main 
tasks will be performed. First, obtain new cross section libraries for nTRACER. Second, apply the methodology used in this work to a full core and compare the results with those obtained with SIMULATE, which is the ICFM nodal code.

\section{ACKNOWLEDGEMENTS}

This work was partly funded by the Swiss Nuclear Safety Inspectorate ENSI (H-101230) and was conducted within the framework of the STARS program (http://www.psi.ch/stars).

\section{REFERENCES}

[1] H. Ferroukhi, K. Hofer, J-M. Hollard, A. Vasiliev, M. A. Zimmermann, "Core Modelling and Analysis of the Swiss Nuclear Power Plants for Qualified R\&D Applications," International Conference on the Physics of Reactors "Nuclear Power: A Sustainable Resource", Interlaken (Switzerland), September 14-19, (2008).

[2] “CASMO5. A FUEL ASSEMBLY BURNUP PROGRAM. User's Manual," Studsvik, SSP07/431 Rev 15.

[3] "SIMULATE5. Advanced Three-Dimensional Multigroup Reactor Analysis Code. User's Manual," Studsvik, SSP-10/438 Rev 10.

[4] “SNF. User's Manual,” Studsvik, SSP-11/328 REV7.

[5] D. Rochman, A. Vasiliev, H. Ferroukhi, M. Pecchia, "Consistent criticality and radiation studies of Swiss spent nuclear fuel: The $\mathrm{CS}_{2} \mathrm{M}$ approach," Journal of Hazardous Materials, 357, pp. 384-392 (2018).

[6] M. Pecchia, H. Ferroukhi, A. Vasiliev, P. Grimm , "Studies of intra-pin power distributions in operated BWR fuel assemblies using MCNP with a cycle check-up methodology," Annals of Nuclear Energy, 129, pp. 67-78 (2019).

[7] J. Leppänen, M. Pusa, T. Viitanen, V. Valtavirta, T. Kaltiaisenaho, "The Serpent Monte Carlo code: Status, development and applications in 2013," Annals of Nuclear Energy, 82, pp. 142-150 (2015).

[8] J.Y. Cho, H.G. Joo, K.S. Kim, and S.Q. Zee, "Three-Dimensional Heterogeneous Whole Core Transport Calculation Employing Planar MOC Solutions," Transactions of the American Nuclear Society, 87, pp. 234-236 (2002).

[9] Y.S. Jung, C.B. Shim, C.H. Lim, H.G. Joo, "Practical numerical reactor employing direct whole core neutron transport and subchannel thermal/hydraulic solvers," Annals of Nuclear Energy, 62, pp. 357-374 (2013).

[10] M. Ryu, Y.S. Jung, H.H. Cho, H.G. Joo, "Solution of the BEAVRS benchmark using the nTRACER direct whole core calculation code," Journal of Nuclear Science and Technology, 52, pp. 961-969 (2015).

[11] A.J. Koning, D.Rochman, "Modern Nuclear Data Evaluation with the TALYS Code System," Nuclear Data Sheets, 113, pp. 2841-2934 (2012).

[12] “The NJOY Nuclear Data Processing System, Version 2016," Los Alamos National Laboratory (LANL), Los Alamos, USA (2017).

[13] O. Cabellos, "Processing of the JEFF-3.2T3 Cross Section Library with the NJOY Code System into Various Formats for Testing Purposes," Nuclear Energy Agency, JEFFDOC1516 (2013). 